\title{
Computational Creativity, Intelligence and Autonomy
}

\author{
J. Mark Bishop · Yasemin J. Erden
}

Received: 11 June 2012/ Accepted: 27 June 2012/Published online: 24 July 2012

(C) Springer Science+Business Media, LLC 2012

The interplay between AI, creativity and autonomy remains an exciting area of research in artificial intelligence and cognitive science. This is evidenced, for example, by the many stimulating papers submitted to and subsequently presented at the recent AISB Computing and Philosophy workshop held at York University in 2011. This Special Issue includes a number of those papers, along with others submitted after the event, all of which investigate one or more of these key concepts within AI. Our contributors come from a wide range of disciplines, and this adds to the rich fecund of material that the issue contains.

We selected these concepts in particular because it is our belief that these three strands are fundamental to the notion of what it is to be human. Alongside ideas about intelligence and autonomy, are also questions about what it is to be creative. For these reasons, issues of "computational creativity" lie at the heart of the intersection of AI, cognitive science, philosophy, and the arts. An autonomous system is typically considered to be a self-determining system, as distinguished from a system whose behaviour is explicitly externally engineered. The concept of autonomy (and autonomous systems) is, therefore, crucial to understanding both intelligent and cognitive systems. In the area of robotics, for instance, issues of embodiment and autonomy are core to the practise of $\mathrm{AI}$ and, in the development of enactivism and emergence, conceptually central to a modern understanding of cognition (an area

J. Mark Bishop $(\square)$

Goldsmiths College London, London, UK

e-mail: m.bishop@gold.ac.uk

Y. J. Erden

St. Mary's University College, London, UK

e-mail: erdenyj@smuc.ac.uk recently explored by Evan Thompson is his 2007 monograph, 'Mind in Life' [1]). Indeed, some commentators such as Maturana and Varela [2] —in their seminal work on autopoiesis and cognition-go so far as to suggest autonomy a necessary hurdle over which any 'living machine' with a claim to genuine teleology must leap.

\section{Synopsis}

To help define the scope of this Special Issue, specifically with respect to the primary issue of autonomy, our opening paper by Vincent Müller (this issue) describes the '2nd European Network for Cognitive Systems, Robotics and Interaction', which took place in October 2011 in Groningen, for which the topic of that meeting was 'Autonomous activity in real-world environments'. As one of the organizers of this event, Müller offers a brief personal report on why autonomy in real-world environments is considered central for cognitive systems research. Key claims identified from that meeting include (a) autonomy is a relative property and a matter of degree, (b) increasing autonomy of an artificial system from its makers and users is a necessary feature of increasingly intelligent systems that can deal with the real-world and (c) more such autonomy means less control but at the same time improved interaction with the system.

Our opening research article by Saunders (this issue) explores the relation between all three strands of our Special Issue by investigating the long-standing debate surrounding the nature of machine intelligence, autonomy and creativity. Within this is offered an argument for the development of autonomous computational creativity that models personal motivations, social interactions and the evolution of domains. From this are discussed different 
approaches to the development of autonomous computational creativity, such as 'artificial creative systems composed of intrinsically motivated agents engaging in language games to interact with a shared social and cultural environment'.

The topic of intelligence receives sustained attention from two of our authors. On the one hand, Schweizer (this issue) responds to typical criticism of the computational theory of mind (CTM) and offers an argument for realization as 'a matter of approximation and degree'. To this, he adds that how we interpret a physical device as performing a computation is in fact 'relative to our purposes and potential epistemic gains', which nevertheless does not 'rule out the possibility of a scientifically defensible account of propositional attitude states in computational terms'. Wiedermann (this issue), on the other hand, tackles controversial issues about the computational power of cognitive systems, the Extended Turing Machine Paradigm and the question of superintelligence. He argues that 'human level intelligence is upper-bounded by the Sigma-2 class of the Arithmetical Hierarchy. In this class, there are problems whose complexity grows faster than any computable function and, therefore, not even exponential growth of computational power can help in solving such problems, or reach the level of superintelligence'.

The issue of creativity inspired diverse thinking from our contributors. Johnson (this issue) notes that computational creativity sometimes involves a search/transformation process, but believes a contrasting approach, whereby search is based on the idea of connotations would be more appropriate. On his account, search processes would be defined according to more exploratory processes, alongside 'a density of connotations'. In the paper by Perlovsky and Levine (this issue), the cognitive bases for creativity are compared with non-creative knowledge acquisition, and 'neural substrates' suggested for these processes. Topics of adaptive behaviour, language and heuristic thinking are considered, alongside functions of 'conceptual, emotional, conscious, and unconscious mechanisms' as pertains to various brain regions. Wiggins (this issue) presents 'a theoretical, hypothetical model of creative cognition, broadly framed within a massively-parallel view of mental computation'. This is based on 'statistical simulation of aspects of memory and perception' and models function rather than detailed mechanism. The crux of the paper lies in the claim that 'the perceptual grounding of the mechanisms presented here may be generalized away, to account, ultimately, for original thought itself'.

The final two papers in this section, shows crossovers between creativity and autonomy. The first by Majid al-Rifaie, Bishop and Caines (this issue) covers the creative potential of Swarm intelligence (SI) algorithms, which the authors claim offers 'a novel integration strategy' in order to sketch 'novel drawings' of an input image. This, they say, exploits 'an artistic tension' between 'birds flocking' and 'ants foraging'. The central concept of the work, the use of Stochastic Diffusion Search to regulate exploration of the entire 'canvas', ensures that the system has 'freedom' to draw at any position on the canvas whilst simultaneously maintaining fidelity to the original concept. The paper concludes by exploring the 'creativity' of this hybrid swarm system in the context of both the 'rhizome' and Deleuze's 'Orchid and Wasp' metaphor. The second paper by d'Inverno and Luck (this issue) explores interconnected ideas of motivation, autonomy and interaction between agents. This is used to defend their claim that creativity can have a central place 'within the design of modern computing systems'. Behaviour in their approach is not prescribed but instead 'determined in relation to motivation', and this forms part of their conclusion that creativity in discovery 'arises from the motivation and autonomy of the individual involved'.

Other papers in our section on autonomy specifically share in common a more theoretical approach to the topic. Kazakov's work (this issue) discusses possible challenges and benefits to developing 'self-cognisant artificial intelligence systems'. 'Greater autonomy', he argues, understood as 'the integration of learning' that would 'allow the artefact to observe its own functionality and build a model of itself' cannot in fact be fully achieved 'without the ability of the learner to model its own performance'. This, he claims, engenders a broad range of implications and issues that the paper then debates. In a similar vein, Magill and Erden (this issue) discuss both the potential and possible limitations to developing truly autonomous cognitive agent systems. Such developments would, they argue, need to take into account the ways in which key terms such as 'desire' are used within applied theoretical models of agency and agent systems. Their argument hangs on the claim that insufficient attention is given to the analysis and clarification of desire as a complex concept (e.g. as distinguished from other mental and motivational states), which this paper seeks to remedy.

We end our SI with a paper that explores autonomy in novel and practical ways and picks up threads of a number of topics raised by the papers in this issue. Froese, Suzuki, Ogai and Ikegami's work (this issue) explores the growing interest of researchers to establish 'a science of the experiential' with regard to the human mind. They propose that this marks a shift from 'cognitive science' to 'consciousness science', which they claim presents 'a profound challenge' to some of the synthetic approaches. Their method seeks to address the challenge of "first-person experience' by designing what they call 'new humancomputer interfaces'. This computer-interface technology would, they suggest, enable them to 'systematically vary 
the ways in which participants experience the world' and thereby allow systematic investigation of the 'mind-as-itcould-be' from the first-person perspective.

\section{Future Research}

The research in this Special Issue contributes to already existing debates within computation, but the benefits of our interdisciplinary approach are that these contributions are often novel, sometimes provocative, and always stimulating. The questions that concepts like intelligence, autonomy and creativity inspire have not been answered in any final way by the work in this issue, nor was this ever the intention of the guest editors. In fact, we sincerely hope to have raised many more questions on these topics than our contributors could ever have hoped to answer, and in this way, to have inspired further work on the topics.

Acknowledgments The guest editors wish to thank all the contributing authors and the anonymous reviewers who helped ensure the quality of this Special Issue.

\section{References}

1. Thompson E. Mind in life: biology, phenomenology, and the sciences of the mind. Cambridge: Harvard University Press; 2007.

2. Maturana HR, Varela FJ. Autopoiesis and cognition: the realization of the living (Boston Studies in the Philosophy of Science), vol. 42. Dordrecht: D. Reidel; 1980. 